M. B. Rai / Our Nature (2003) 1: 42-48

\title{
Medicinal Plants of Tehrathum District, Eastern Nepal
}

\author{
Mani Bahadur Rai* \\ Department of Botany, P. G. Campus, Biratnagar
}

\begin{abstract}
Vaidhya (Herbalist) use the medicinal plants to remove the ailments since time immemorial. Tehrathum district is found rich in medicinal plants. A total of 105 vascular plants were recorded to be used in different diseases. Information was collected by discussion through local communities and field observations. Some plants were found to be used in different diseases in different places. The indigenous knowledge is transferring from generation to generation in certain families without any written records.
\end{abstract}

Keywords: Medicinal Plants, NTFPs, Tehrathum, Shaman

\section{Introduction}

Tehrathum is a hilly district, located $76^{0} 25^{\prime} \mathrm{E}$ to $87^{\circ} 75^{\prime} \mathrm{E}$ and $26^{\circ} 59^{\prime} \mathrm{N}$ to $27^{\circ} 30^{\prime} \mathrm{N}$ longitude and latitude of eastem Nepal, covering 679 sq. km. area. The altitude ranges from 530m (Tamor Phedi') to 3,010m ('Tinjure') (Rai 1996). The area covers subtropical and temperate climate. The major ethnic groups of the district are Limbu, Sherpa, Tamang, Rai, Magar, Gurung, and Newar. Amomum aromaticum, Asparagus spp., Astilbe rivularis, Bergenia ciliata, Daphne spp., Lycopodium clavatum, Nardostachys grandiflora, Picrorhiza scrophulariiflora, Piper longum, Potentilla fulgen, Rubia manjith, Sapindus mukorossi, Swertia chirayita, Taxus baccata, Thysanolaena maxima, Zanthoxylum armatum, Zingeber officinale are exported as the main non timber forest product (NTFPs) from the district.

Most of the people of Tehrathum inhabit in rural area. The forest plants, as other areas, play an important part in their life and derive food, fodder, fuel, etc from their surroundings. They are also familiar about medicinal plants. Especially local 'Vaidhya' and 'Dhami-Jhankri' (Shaman) know the uses of these plants and are practicing to remove the ailments since time immemorial. So, medicinal

Correspondence: *Birat Science Campus, Biratnagar plants are their main source of remedy for diseases. Those persons, who use these plants, often keep their knowledge secret. They are against the exploration of their traditional knowledge. Such knowledge is transferring from generation to generation in certain families without any written record.

Works on medicinal plant surveys have been done from eastern Nepal by several workers (Banerji 1957, Manandhar 1971, Dobremez 1976, Gautam 1995). However, the literature of medicinal plants from Tehrathum district is not available except a single report on NTFPs (Pradhan and Manandhar 1997). Present work is a detailed survey of vascular medicinal plants, with help of local experts of the district. The surveying work was done mainly in five Village Development Committees. These are Jaljale, Simle, Oyakjung, Esibu and Solma. Plants were collected from forests of Chichiling (Jaljale and Simle VDCs), Sikre (adjoining area of Oyakjung, Samdhu and Pauthak VDCs) and Tinjure (Solma, Sungnam and Ambung). Most of such plants were found in temperate climate.

\section{Materials and Methods}

Local 'Dhami-Jhakri' and 'Vaidhya' were identified. By making good relation with them, 
M. B. Rai / Our Nature (2003) 1: 42-48

information about medicinally using plants in different diseases were recorded. Thereafter, they were also requested to involve in the field visit during 1996.

The herbarium specimens were identified with the help of available literature (Hookers 1872-1897, Hains 1961, Grieson and Long 1983, Anonymous 1986, Mabberley 1987, Davis and Cullen 1988, Siwakoti 1995). The voucher specimens are deposited in the Department of Botany, Post Graduate Campus, Biratnagar. The recorded specimens have been arranged in alphabetical order with botanical and vernacular name after their family. The parts used along with their value also described.

\section{Results and Discussion}

A total of 105 species of vascular plants were found frequently used in Tehrathum district in different diseases (Table 1). Some of these were found to be used in more than one disease. A particular plant used for a specific disease in one places was found to be used for a different diseases in another places although the plants were available in both places. For example, Cassia fistula is used in diuretic, purgative and rheumatism at Jaljale, while in Solma; the plant is used as a remedy's for upper respiratory diseases. 'Vaidhyas' were found having adequate information about medicinal plant. They use these plants for curing diseases. 'Dhami-Jhankri' also do the same but they give priority to tantra, mantra. The work is totally based on the information provided by the local 'Vaidhyas' and 'Dhami-Jhankri.'

Table 1. List of Medicinal Plants

\begin{tabular}{|c|l|l|l|l|l|}
\hline S. N. & \multicolumn{1}{|c|}{ Family } & Botanical Name & $\begin{array}{c}\text { Vernacular } \\
\text { Name }\end{array}$ & \multicolumn{1}{|c|}{$\begin{array}{c}\text { Parts } \\
\text { used }\end{array}$} & \multicolumn{1}{|c|}{ Diseases } \\
\hline 1 & Leguminosae & Abrus precatoris & Lalgedi & $\begin{array}{l}\text { Seed, leaf, } \\
\text { root }\end{array}$ & $\begin{array}{l}\text { Nervous disorder, } \\
\text { eye, skin diseases. }\end{array}$ \\
\hline 2 & $"$ & Acacia catechu & Khayar & Wood & $\begin{array}{l}\text { Astringent, cooling, } \\
\text { digest }\end{array}$ \\
\hline 3 & Amaranthaceae & $\begin{array}{l}\text { Achyranthes } \\
\text { aspera }\end{array}$ & Apamarga & Root & $\begin{array}{l}\text { Stones, diarrhea, } \\
\text { dysentery }\end{array}$ \\
\hline 4 & Araceae & Acorus calamus & Bojo & Rhizome & $\begin{array}{l}\text { Cough, whooping } \\
\text { cough, bronchitis }\end{array}$ \\
\hline 5 & Acanthaceae & Adhatoda vasica & Asuro & $\begin{array}{l}\text { All parts } \\
\text { except } \\
\text { stem }\end{array}$ & $\begin{array}{l}\text { Diarrhea, dysentery } \\
\text { fruit }\end{array}$ \\
\hline 7 & Asteraceae & $\begin{array}{l}\text { Ageratum } \\
\text { conyzoides }\end{array}$ & Ilame jhar & $\begin{array}{l}\text { Leaf, young } \\
\text { shoot }\end{array}$ & $\begin{array}{l}\text { Control bleeding } \\
\text { (wound) }\end{array}$ \\
\hline 8 & Liliaceae & Allium cepa & Piyas & Bulb & $\begin{array}{l}\text { Aphrodisiac, anti- } \\
\text { malaria }\end{array}$ \\
\hline 9 & " & A. sativa & Lasun & Bulb & Gastric, diabetes \\
\hline 10 & " & Aloe barbadensis & $\begin{array}{l}\text { Gheu } \\
\text { Kumari }\end{array}$ & Leaf & Burns \\
\hline
\end{tabular}


M. B. Rai / Our Nature (2003) 1: 42-48

\begin{tabular}{|c|c|c|c|c|c|}
\hline 11 & Zingeberaceae & $\begin{array}{l}\text { Amomum } \\
\text { subulatum }\end{array}$ & Alaichi & $\begin{array}{l}\text { Fruit and } \\
\text { seed }\end{array}$ & $\begin{array}{l}\text { Indigestion, vomiting, } \\
\text { aphrodisiac }\end{array}$ \\
\hline 12 & Annonaceae & $\begin{array}{l}\text { Annona } \\
\text { squamosa }\end{array}$ & Sarifa & Fruit & $\begin{array}{l}\text { Digestive, vomiting, } \\
\text { diarrhea, dysentery }\end{array}$ \\
\hline 13 & Euphorbiaceae & $\begin{array}{l}\text { Antidesma } \\
\text { diandrum }\end{array}$ & Archal & $\begin{array}{l}\text { Leaves, } \\
\text { bark, root }\end{array}$ & $\begin{array}{l}\text { Astringent, cholera \& } \\
\text { dysentery }\end{array}$ \\
\hline 14 & Asteraceae & $\begin{array}{l}\text { Artemisia } \\
\text { vulgaris }\end{array}$ & Titepati & Root & $\begin{array}{l}\text { Anathematic, nervous } \\
\text { fresher, antispasmodic }\end{array}$ \\
\hline 15 & Saxifragaceae & Astilbe rivularis & $\begin{array}{l}\text { Thulo } \\
\text { Okhati }\end{array}$ & Rhizome & $\begin{array}{l}\text { Uterine contraction } \\
\text { during birth, dysentery, }\end{array}$ \\
\hline 16 & Moraceae & $\begin{array}{l}\text { Artocarpus } \\
\text { heterophyllus }\end{array}$ & Katahar & $\begin{array}{l}\text { Leaves, } \\
\text { root }\end{array}$ & $\begin{array}{l}\text { Skin diseases, } \\
\text { diarrhea }\end{array}$ \\
\hline 17 & Liliaceae & $\begin{array}{l}\text { Asparagus } \\
\text { racemosus }\end{array}$ & Kurilo & $\begin{array}{l}\text { Rhizome } \\
\text { bulb }\end{array}$ & $\begin{array}{l}\text { Galactagogue, } \\
\text { aphrodisiac, diabetes }\end{array}$ \\
\hline 18 & Leguminosae & Bauhinia vahlli & Bhorla & $\begin{array}{l}\text { Seeds, } \\
\text { young } \\
\text { shoot }\end{array}$ & Snake bite, aphrodisiac \\
\hline 19 & $"$ & B. variegata & Koiralo & $\begin{array}{l}\text { Root, } \\
\text { bark, bud }\end{array}$ & $\begin{array}{l}\text { Dyspepsia, obesity, } \\
\text { dysentery, cough, piles }\end{array}$ \\
\hline 20 & Begonaceae & Begonia rubella & Makarkachi & Leaves & $\begin{array}{l}\text { Chest pain, } \\
\text { antibacterial }\end{array}$ \\
\hline 21 & Berberidaceae & Berberis aristata & Chutro & Fruit & $\begin{array}{l}\text { Jaundice, malaria, } \\
\text { piles, sores, eye } \\
\text { diseases }\end{array}$ \\
\hline 22 & Saxifragaceae & Bergenia ciliata & Pakhanbet & Rhizome & $\begin{array}{l}\text { Uterine contraction, } \\
\text { ulcers, fever, kidney } \\
\text { stone }\end{array}$ \\
\hline 23 & Bombaceae & Bombax ceiba & Simal & Bark & $\begin{array}{l}\text { Demulcent, tonic, } \\
\text { dysentery, skin } \\
\text { eruptions }\end{array}$ \\
\hline 24 & Logaginaceae & $\begin{array}{l}\text { Buddleja } \\
\text { asiatica }\end{array}$ & Bhimsenpate & $\begin{array}{l}\text { Root, } \\
\text { leaves }\end{array}$ & Malaria, tumor \\
\hline 25 & Verbenaceae & $\begin{array}{l}\text { Callicarpa } \\
\text { macrophylla }\end{array}$ & Dahidangalo & $\begin{array}{l}\text { Root, } \\
\text { leaves }\end{array}$ & $\begin{array}{l}\text { Stomach disorder, } \\
\text { rheumatism. }\end{array}$ \\
\hline 26 & Asclepidaceae & $\begin{array}{l}\text { Calotropis } \\
\text { gigantea }\end{array}$ & Aank & $\begin{array}{l}\text { Root, } \\
\text { leaves }\end{array}$ & $\begin{array}{l}\text { Cold, coughs, asthma, } \\
\text { dysentery, } \\
\text { elephantiasis }\end{array}$ \\
\hline 27 & Cannabinaceae & Cannabis sativa & Ganja & $\begin{array}{l}\text { Leaves, } \\
\text { seeds }\end{array}$ & $\begin{array}{l}\text { Diarrhea, sedative, } \\
\text { narcotize }\end{array}$ \\
\hline 28 & Leguminosae & Cassia fistula & Rajbrikhchha & $\begin{array}{l}\text { Fruit, } \\
\text { seeds }\end{array}$ & $\begin{array}{l}\text { Diuretic, purgative, } \\
\text { rheumatism, } \\
\text { respiratory diseases }\end{array}$ \\
\hline 29 & Apiaceae & Centella asiatica & Ghod tapre & Shoot & $\begin{array}{l}\text { Diuretic, blood } \\
\text { purifier, leprosy }\end{array}$ \\
\hline 30 & Menispermaceae & $\begin{array}{l}\text { Cissampelis } \\
\text { pareira }\end{array}$ & Gugarganu & $\begin{array}{l}\text { Root, } \\
\text { leaves }\end{array}$ & $\begin{array}{l}\text { Diuretic, purgative, } \\
\text { dyspepsia, diarrhea, } \\
\text { itch }\end{array}$ \\
\hline
\end{tabular}


M. B. Rai / Our Nature (2003) 1: 42-48

\begin{tabular}{|c|c|c|c|c|c|}
\hline 31 & Rutaceae & Citrus lemon & Kagati & Fruit & $\begin{array}{l}\text { Carminative, } \\
\text { rheumatism } \\
\text { dysentery, diarrhea }\end{array}$ \\
\hline 32 & Lamiaceae & $\begin{array}{l}\text { Clinopodium } \\
\text { umbrasum }\end{array}$ & Tulsijhar & Leaves & Astringent \\
\hline 33 & Poaceae & $\begin{array}{l}\text { Coix lachryma- } \\
\text { jobi }\end{array}$ & Bhirkoule & Fruit, root & $\begin{array}{l}\text { Diuretic, blood } \\
\text { purifier \& menstrual } \\
\text { disorder }\end{array}$ \\
\hline 34 & Cucurbitaceae & Cucurbita pepo & Pharsi & Seed & Anathematic \\
\hline 35 & Zingeberaceae & Curcuma longa & Kalo Besar & Rhizome & $\begin{array}{l}\text { Common cold, cough, } \\
\text { skin diseases }\end{array}$ \\
\hline 36 & Cuscutaceae & Cuscuta reflexa & Akash beli & Shoot & $\begin{array}{l}\text { Jaundice, urinary tract } \\
\text { inflammation }\end{array}$ \\
\hline 37 & Solanaceae & Datura metel & Kalo Daturo & $\begin{array}{l}\text { Leaves, } \\
\text { flowers, } \\
\text { fruit }\end{array}$ & $\begin{array}{l}\text { Mad dog bite, sores, } \\
\text { dandruff, hair falling }\end{array}$ \\
\hline 38 & Poaceae & $\begin{array}{l}\text { Desmostachya } \\
\text { bipinata }\end{array}$ & Kush & Rhizome & Diuretic, dysentery \\
\hline 39 & $\begin{array}{l}\text { Hydrangeacea } \\
\text { e }\end{array}$ & $\begin{array}{l}\text { Dichroa } \\
\text { febrifuga }\end{array}$ & Basak & $\begin{array}{l}\text { Root, } \\
\text { leaves }\end{array}$ & Malaria fever \\
\hline 40 & Liliaceae & $\begin{array}{l}\text { Disporum } \\
\text { cantoniense }\end{array}$ & Aklebir & Root & Headache, fever \\
\hline 41 & Caryophyllaceae & $\begin{array}{l}\text { Drymaria } \\
\text { cordata }\end{array}$ & Abijalo & Leaves & $\begin{array}{l}\text { Laxative, diarrhea, } \\
\text { dysentery }\end{array}$ \\
\hline 42 & Elaeocarpaceae & $\begin{array}{l}\text { Elaeocarpus } \\
\text { sphaericus }\end{array}$ & Rudrakshya & Fruit & $\begin{array}{l}\text { Headache, epileptic } \\
\text { fits }\end{array}$ \\
\hline 43 & Asteraceae & Eclipta alba & Bhumiraj & $\begin{array}{l}\text { Whole } \\
\text { plant }\end{array}$ & $\begin{array}{l}\text { Emetic, purgative, } \\
\text { jaundice }\end{array}$ \\
\hline 44 & Euphorbiaceae & $\begin{array}{l}\text { Emblica } \\
\text { officinalis }\end{array}$ & Amala & Bark, fruit & $\begin{array}{l}\text { Gastric trouble, } \\
\text { haemorrhage, } \\
\text { jaundice, dyspepsia }\end{array}$ \\
\hline 45 & Moraceae & $\begin{array}{l}\text { Ficus } \\
\text { bengalensis }\end{array}$ & Bar & $\begin{array}{l}\text { Leaves, } \\
\text { barks }\end{array}$ & $\begin{array}{l}\text { Rheumatism, } \\
\text { dysentery, diabetes, } \\
\text { vomiting }\end{array}$ \\
\hline 46 & $"$ & F. cunia & Khanyu & Root, fruit & $\begin{array}{l}\text { Aphthous complaints, } \\
\text { bladder ailments }\end{array}$ \\
\hline 47 & $"$ & F. lacor & Kabhro & Bark & $\begin{array}{l}\text { Ulcer, gargle in } \\
\text { salivation }\end{array}$ \\
\hline 48 & $"$ & F. religiosa & Pipal & $\begin{array}{l}\text { Bark, } \\
\text { leaves }\end{array}$ & $\begin{array}{l}\text { Ulcer, skin diseases, } \\
\text { antibacterial, purgative }\end{array}$ \\
\hline 49 & Leguminosae & Glycine $\max$ & Bhatmas & Seed & Diabetes \\
\hline 50 & Apiaceae & $\begin{array}{l}\text { Heracleum } \\
\text { nepalense }\end{array}$ & Chingfing & Fruit & Common cold, cough \\
\hline 51 & Meliaceae & Heynea trijuga & Ankhataruwa & Leaves & Cholera, burn \\
\hline 52 & Apocynaceae & $\begin{array}{l}\text { Holorrhena } \\
\text { antidysentrica }\end{array}$ & Khirro & Bark, seed & $\begin{array}{l}\text { Astringent, dysentery, } \\
\text { anathematic, } \\
\text { febrifugal }\end{array}$ \\
\hline
\end{tabular}


M. B. Rai / Our Nature (2003) 1: 42-48

\begin{tabular}{|c|c|c|c|c|c|}
\hline 53 & Saururaceae & $\begin{array}{l}\text { Houttuynia } \\
\text { cordata }\end{array}$ & Gane & $\begin{array}{l}\text { Whole } \\
\text { plant }\end{array}$ & $\begin{array}{l}\text { Cooling indigestion, } \\
\text { dysentery, diuretic }\end{array}$ \\
\hline 54 & Poaceae & $\begin{array}{l}\text { Imperata } \\
\text { cylindrica }\end{array}$ & Siru & Rhizome & $\begin{array}{l}\text { Piles, diarrhea, } \\
\text { dysentery, gonorrhea, } \\
\text { sedative }\end{array}$ \\
\hline 55 & Junglandaceae & Juglans regia & Okhar & $\begin{array}{l}\text { Leaves, } \\
\text { fruits }\end{array}$ & $\begin{array}{l}\text { Bactericide, } \\
\text { rheumatism, laxative }\end{array}$ \\
\hline 56 & Brassicaceae & $\begin{array}{l}\text { Lepidium } \\
\text { sativum }\end{array}$ & Chamsur & $\begin{array}{l}\text { Leave, } \\
\text { fruit }\end{array}$ & $\begin{array}{l}\text { Asthma, cough, piles, } \\
\text { diuretic }\end{array}$ \\
\hline 57 & Lauraceae & Lindera neesiana & Siltimur & Fruit & $\begin{array}{l}\text { Carminative, } \\
\text { headache, gastric } \\
\text { trouble }\end{array}$ \\
\hline 58 & Lycododiaceae & $\begin{array}{l}\text { Lycopodium } \\
\text { clavatum }\end{array}$ & Nagbeli & Fruit & $\begin{array}{l}\text { Scabies, rheumatism, } \\
\text { catarrh, malaria }\end{array}$ \\
\hline 59 & Ericaceae & Lyonia avalifolia & Angeri & Leaves & Skin diseases \\
\hline 60 & Sapotaceae & $\begin{array}{l}\text { Madhuca } \\
\text { butyracea }\end{array}$ & Chiuri & Fruit & $\begin{array}{l}\text { Rheumatism, } \\
\text { emollient }\end{array}$ \\
\hline 61 & $"$ & M. lougifolia & Mauwa & Flower & $\begin{array}{l}\text { Coughs, colds, } \\
\text { bronchitis }\end{array}$ \\
\hline 62 & Myrsinaceae & $\begin{array}{l}\text { Maesa } \\
\text { macrophylla }\end{array}$ & Bhogote & $\begin{array}{l}\text { Young } \\
\text { shoot }\end{array}$ & $\begin{array}{l}\text { Anathematic, } \\
\text { dysentery, gastric } \\
\text { trouble }\end{array}$ \\
\hline 63 & Berberidaceae & $\begin{array}{l}\text { Mahonia } \\
\text { napaulensis }\end{array}$ & Chutro & Fruit & Diuretic, dysentery \\
\hline 64 & Lamiaceae & Mentha arvensis & Pudin & Shoot & $\begin{array}{l}\text { Cholera, anathematic, } \\
\text { cough }\end{array}$ \\
\hline 65 & Leguminosae & Mimosa pudica & Lajjawati & $\begin{array}{l}\text { Root, } \\
\text { leaves }\end{array}$ & $\begin{array}{l}\text { Urinary complaints, } \\
\text { swelling, sores, piles }\end{array}$ \\
\hline 66 & $"$ & Mucuna purita & Kauso & Root & $\begin{array}{l}\text { Tonic, stimulant, } \\
\text { diuretic, purgative }\end{array}$ \\
\hline 67 & Musaceae & Musa paradisica & Kera & Fruit & $\begin{array}{l}\text { Diarrhea, dysentery, } \\
\text { diabetes }\end{array}$ \\
\hline 68 & Myricaceae & Myrica esculenta & Kaphal & Bark & $\begin{array}{l}\text { Asthma, bronchitis, } \\
\text { dysentery, diuretic }\end{array}$ \\
\hline 69 & Lamiaceae & $\begin{array}{l}\text { Ocimum } \\
\text { basilicum }\end{array}$ & $\begin{array}{l}\text { Babari } \\
\text { Phool }\end{array}$ & Leaves & $\begin{array}{l}\text { Carminative, diuretic, } \\
\text { gonorrhea, dysentery }\end{array}$ \\
\hline 70 & " & O. sanctum & Tulsi & Leaves & $\begin{array}{l}\text { Diaphoretic, } \\
\text { stimulating, expectorant }\end{array}$ \\
\hline 71 & Bignoniaceae & $\begin{array}{l}\text { Oroxylum } \\
\text { indicum }\end{array}$ & Totola & $\begin{array}{l}\text { Bark, } \\
\text { leaves }\end{array}$ & $\begin{array}{l}\text { Rheumatism, ulcer, } \\
\text { diarrhea, dysentery }\end{array}$ \\
\hline 72 & Santalaceae & $\begin{array}{l}\text { Osyris } \\
\text { wightiana }\end{array}$ & Haadjaro & $\begin{array}{l}\text { Roots, } \\
\text { leaves }\end{array}$ & $\begin{array}{l}\text { Bone crack, } \\
\text { contraction of uterine } \\
\text { muscles. }\end{array}$ \\
\hline 73 & Oxalidaceae & $\begin{array}{l}\text { Oxalis } \\
\text { corniculata }\end{array}$ & Chariamilo & Shoot & $\begin{array}{l}\text { Astringent, febrifuge, } \\
\text { antiseptic }\end{array}$ \\
\hline 74 & Piperaceae & Piper nigrum & Marich & Fruit & Common cold, catarrh \\
\hline
\end{tabular}


M. B. Rai / Our Nature (2003) 1: 42-48

\begin{tabular}{|c|c|c|c|c|c|}
\hline 75 & $"$ & P. peepuloids & Pipla & Fruit & $\begin{array}{l}\text { Common cold, } \\
\text { leprosy }\end{array}$ \\
\hline 76 & Plumbagonaceae & $\begin{array}{l}\text { Plumbago } \\
\text { zeylanica }\end{array}$ & Chittu & Leaves & $\begin{array}{l}\text { Leprosy, skin } \\
\text { diseases, bactericide, } \\
\text { fungicide }\end{array}$ \\
\hline 77 & Lamiaceae & $\begin{array}{l}\text { Pogostemon } \\
\text { benghalensis }\end{array}$ & Rudilo & $\begin{array}{l}\text { Root, } \\
\text { leaves }\end{array}$ & Haemorrhage \\
\hline 78 & Polygonaceae & $\begin{array}{l}\text { Polygonum } \\
\text { molle }\end{array}$ & Thotne & $\begin{array}{l}\text { Young } \\
\text { shoot }\end{array}$ & Astringent \\
\hline 79 & Rosaceae & $\begin{array}{l}\text { Potentilla } \\
\text { fulgens }\end{array}$ & Bajradanti & Root & $\begin{array}{l}\text { Diarrhea, gastric } \\
\text { trouble }\end{array}$ \\
\hline 80 & Myrtaceae & Psidium guajava & Ambak & $\begin{array}{l}\text { Bark, leaf, } \\
\text { fruit }\end{array}$ & $\begin{array}{l}\text { Cholera, vomiting, } \\
\text { diarrhea, dysentery }\end{array}$ \\
\hline 81 & Punicaceae & Punica granatum & Anar & $\begin{array}{l}\text { Bark, leaf, } \\
\text { fruit }\end{array}$ & Dysentery, bronchitis \\
\hline 82 & $"$ & Rabdosia coesta & Mirre & $\begin{array}{l}\text { Young } \\
\text { shoot }\end{array}$ & Antiseptic \\
\hline 83 & Apocynaceae & $\begin{array}{l}\text { Rauwolfia } \\
\text { serpentina }\end{array}$ & $\begin{array}{l}\text { Chandmaru } \\
\text { wa }\end{array}$ & $\begin{array}{l}\text { Bark, leaf, } \\
\text { fruit }\end{array}$ & $\begin{array}{l}\text { Mental disorder, } \\
\text { blood pressure, } \\
\text { diabetes }\end{array}$ \\
\hline 84 & Ericaceae & $\begin{array}{l}\text { Rhododendron } \\
\text { arboreum }\end{array}$ & Laligurans & Flower & Diarrhea, dysentery \\
\hline 85 & Anacardiaceae & Rhus parviflora & Satibayar & Fruit & $\begin{array}{l}\text { Diarrhea, dysentery, } \\
\text { stomached }\end{array}$ \\
\hline 86 & Rubiaceae & Rubia cordifolia & Majito & $\begin{array}{l}\text { Root, } \\
\text { leaves }\end{array}$ & $\begin{array}{l}\text { Ulcers, } \\
\text { inflammations, skin } \\
\text { diseases }\end{array}$ \\
\hline 87 & Rosaceae & Rubus ellipticus & Ainselu & Root, bark & $\begin{array}{l}\text { Wounds, gastric } \\
\text { troubles, diarrhea, } \\
\text { dysentery }\end{array}$ \\
\hline 88 & Sapindaceae & $\begin{array}{l}\text { Sapindus } \\
\text { cytheria }\end{array}$ & Amaro & $\begin{array}{l}\text { Flower, } \\
\text { fruit }\end{array}$ & Pneumonia \\
\hline 89 & $"$ & S. mukorossi & Rittha & Fruits & Salivation, epilepsy \\
\hline 90 & Theaceae & Schima wallichii & Chilaune & $\begin{array}{l}\text { Bark, } \\
\text { leaves }\end{array}$ & Rubefacient, fever \\
\hline 91 & Scrophulariaceae & Scoparia dulcis & Chini Jhar & $\begin{array}{l}\text { Root, } \\
\text { leaves }\end{array}$ & $\begin{array}{l}\text { Fever, cough, } \\
\text { bronchitis, } \\
\text { menstruation disorder }\end{array}$ \\
\hline 92 & Apiaceae & $\begin{array}{l}\text { Selinum } \\
\text { tenuifolium }\end{array}$ & Bhutkesh & Fruit & $\begin{array}{l}\text { Aphrodisiac, sedative, } \\
\text { rheumatism }\end{array}$ \\
\hline 93 & $\begin{array}{l}\text { Diptherocarpa } \\
\text { ceae }\end{array}$ & Shorea robusta & Sal & $\begin{array}{l}\text { Resin, } \\
\text { fruit }\end{array}$ & $\begin{array}{l}\text { Astringent, diarrhea, } \\
\text { dysentery }\end{array}$ \\
\hline 94 & Smilacaceae & $\begin{array}{l}\text { Smilax } \\
\text { macrophylla }\end{array}$ & Kukurdaino & Root & $\begin{array}{l}\text { Rheumatism, } \\
\text { bloodless dysentery }\end{array}$ \\
\hline 95 & Solanaceae & $\begin{array}{l}\text { Solanum } \\
\text { tuberosum }\end{array}$ & Alu & $\begin{array}{l}\text { Leaves, } \\
\text { tuber }\end{array}$ & $\begin{array}{l}\text { Antispasmodic in } \\
\text { cough, burn }\end{array}$ \\
\hline
\end{tabular}


M. B. Rai / Our Nature (2003) 1: 42-48

\begin{tabular}{|c|l|l|l|l|l|}
\hline 96 & Asteraceae & Spilanthes calva & $\begin{array}{l}\text { Pur-pure } \\
\text { jhar }\end{array}$ & Flower & $\begin{array}{l}\text { Toothache, throat } \\
\text { pain, headache }\end{array}$ \\
\hline 97 & Gentinaceae & $\begin{array}{l}\text { Swertia } \\
\text { chirayita }\end{array}$ & Chirayito & $\begin{array}{l}\text { Whole } \\
\text { plant }\end{array}$ & $\begin{array}{l}\text { Hypertension, } \\
\text { diabetes }\end{array}$ \\
\hline 98 & Asteraceae & Tagetes petula & Sayapatri & $\begin{array}{l}\text { Leaves, } \\
\text { flower }\end{array}$ & $\begin{array}{l}\text { Purgative, } \\
\text { carminative, } \\
\text { antiseptic, jaundice }\end{array}$ \\
\hline 100 & Polygonaceace & $\begin{array}{l}\text { Viscum } \\
\text { articulatum }\end{array}$ & Hadachur & Shoot & $\begin{array}{l}\text { Whole } \\
\text { plant } \\
\text { expectorant, blood } \\
\text { purifier. }\end{array}$ \\
\hline 101 & Verbenaceae & Vitex negundo & Shimali & $\begin{array}{l}\text { Leaves, } \\
\text { flower }\end{array}$ & $\begin{array}{l}\text { Cancer, diarrhea, liver } \\
\text { complaints }\end{array}$ \\
\hline 102 & Lyrthaceae & $\begin{array}{l}\text { Woodfordia } \\
\text { fructicosa }\end{array}$ & Dhaiaro & Flower & $\begin{array}{l}\text { Ulcers, wounds, } \\
\text { cough }\end{array}$ \\
\hline 103 & Rutaceae & $\begin{array}{l}\text { Zanthoxylum } \\
\text { armatum }\end{array}$ & Timur & $\begin{array}{l}\text { Bark, leaf, } \\
\text { fruit }\end{array}$ & $\begin{array}{l}\text { Stomached, } \\
\text { trouble }\end{array}$ \\
\hline 104 & Zingeberaceae & $\begin{array}{l}\text { Zingiber } \\
\text { officinale }\end{array}$ & Aduwa & Rhizome & $\begin{array}{l}\text { Expectorant, } \\
\text { carminative, } \\
\text { digestive, coughs. }\end{array}$ \\
\hline 105 & Rhamnaceae & $\begin{array}{l}\text { Zizyphus } \\
\text { mauritiana }\end{array}$ & Bayar & Root, seed & $\begin{array}{l}\text { Pneumonia, blood } \\
\text { purifier }\end{array}$ \\
\hline
\end{tabular}

\section{Acknowledgements}

Author is grateful to Mr. Rajendra Prasad Shah (Research Guide and Thesis Adviser, Lecturer of MMAC, Biratnagar). Author would like to thank Mr. Sher Bahadur Singhak (Vaidhya, Jaljale VDC-5, Tehrathum) for his kind co-operation

\section{References}

Anonymous 1986. Flora of Kathmandu Valley. Bull. Dept. Med. Pl. Nep. No. 11, Kathmandu.

Banerji, M. L. 1957. Some Edible and Medicinal Plants From East Nepal. J. Bomb. Nat. Hist. 53: 153-155.

Davis, P. H. and J. Cullen 1988. The Identification of Flowering Plant Families. Edinburgh.

Dobremez, J. F. 1967. Exploitation and Prospects of Medicinal Plants in Eastern Nepal. Mountain Environment and Development. Kathmandu.SATA (Swiss Association for Technical Assistance), pp. 97-110.

Gautam, T. P. 1995. Medicinal Plants of Panchthar District. M. Sc. Thesis. Department of Botany, P. G. Campus, Biratnagar.

Grierson, A. J. C. and D. G. Long 1983. Flora of Bhutan. VolsI-II, Edinburgh.
Hains, H. H. 1961. The Botany of Bihar and Orisa. Allahabad.

Hooker, J. D. 1872-97. Flora of British India. Vols. I-VII, London.

Mabberley, D. J. 1987. The Plant-Book. A Portable Dictionary of the Vascular Plants. Cambridge University Press, Cambridge.

Manandhar, N. P. 1971. Some Medicinal Plants of Eastern Nepal. The Rising Nepal. March 18, 1971, pp.2.

Pradhan, M. and V. Manandhar 1997. A Survey of Economically Utilized NTFPs in Tehrathum District. A report submitted on workshop of ANSAB, Kathmandu, Nepal.

Rai, M. B. 1996. Medicinal Plants of Tehrathum District, Eastern Nepal. M. Sc. thesis, P. G. Campus, T. U.

Siwakoti, M. 1995. Flora of Plain region of Eastern Nepal. Biratnagar-Dharan, (Ph. D.thesis) T. M. Bhagalpur, University. 